
\title{
$\begin{array}{ll}\text { Research Square } & \begin{array}{l}\text { Preprints are preliminary reports that have not undergone peer review. } \\ \text { They should not be considered conclusive, used to inform clinical practice, } \\ \text { or referenced by the media as validated information. }\end{array}\end{array}$ \\ Formation of autotriploid Carassius auratus and its fertility-related genes analysis
}

\section{Chongqing Wang}

Hunan Normal University

Xiang Luo

Hunan Normal University

Huan Qin

Hunan Normal University

Chun Zhao

Hunan Normal University

Li Yang

Hunan Normal University

Tingting Yu

Hunan Normal University

\section{Yuxin Zhang}

Hunan Normal University

\section{Xu Huang}

Hunan Normal University

\section{Xidan Xu}

Hunan Normal University

Qinbo Qin ( $\nabla$ qqb@hunnu.edu.cn )

Hunan Normal University

Shaojun Liu

Hunan Normal University

\section{Research Article}

Keywords: fertility, autotriploid fish, transcriptome, gonad

Posted Date: April 13th, 2021

DOI: https://doi.org/10.21203/rs.3.rs-397574/v1

License: (9) (1) This work is licensed under a Creative Commons Attribution 4.0 International License. Read Full License 


\section{Abstract \\ Background}

Formation of triploid organism is useful in genetics and breeding. In this study, autotriploid Carassius

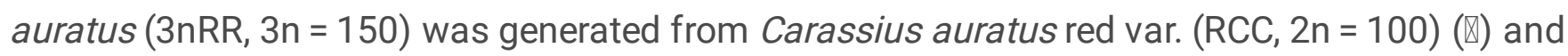
autotetraploid Carassius auratus ( $4 n R R, 4 n=200)(\mathbb{~})$. The female $3 n R R$ produced haploid, diploid and triploid eggs, whereas the male $3 n R R$ was infertile. The aim of the present study was to explore fertility of potential candidate genes of $3 \mathrm{nRR}$.

\section{Results}

Gonadal transcriptome profiling of four groups ( 3 females RCC (FRCC), 3 males 4nRR (M4nRR), 3 males 3nRR (M3nRR) and 3 females 3nRR (F3nRR)) was performed using RNA-SEq. A total of $78.90 \mathrm{~Gb}$ of clean short reads and 24,262 differentially expressed genes (DEGs), including 20,155 in F4nRR vs FRCC and 4,107 in M3nRR vs M4nRR were identified. A total of 106 enriched pathways were identified through KEGG enrichment analysis. Out of the enriched pathways, 44 and 62 signalling pathways were identified in F3nRR vs FRCC and M3nRR vs M4nRR, respectively. A total of 80 and 25 potential candidate genes for fertility-related in F3nRR and M3nRR were identified, through GO, KEGG analyses and the published literature. Moreover, protein-protein interaction (PPI) network construction of these fertility-associated genes were performed. Analysis of the PPI networks showed that 6 hub genes (MYC, SOX2, BMP4, GATA4, PTEN and BMP2) were involved in female fertility of F3nRR, and 2 hub genes (TP53 and FGF2) were involved in male sterility of M3nRR.

\section{Conclusions}

Establishment of autotriploid fish offers an ideal model to study reproductive traits of triploid fish. Further, RNA-Seq data revealed 8 hub genes related to fertility in 3nRR. We further identified 6 genes, namely, MYC, SOX2, BMP4, GATA4, PTEN and BMP2, involved in the female fertility of the F3nRR. Moreover, 2 genes, namely, TP53 and FGF2, were related to the male sterility of the M3nRR. These findings provide information on reproduction and breeding in triploid fish.

\section{Background}

Polyploid organisms have three or more chromosome sets. Triploidy, an example of polyploids, plays a vital role in the process of biological evolution and can be divided into autotriploidy and allotriploidy [1]. Allotriploids have three chromosome sets from two or more different species, whereas autotriploids have three chromosome sets derived from a single taxon. 
Development of gonads is critical to fertility in sexually reproducing organisms especially in triploids and is tightly regulated by complex processes [2]. Sex determination, sexual differentiation and gametogenesis are important processes during gonadal development. Any abnormality in these events can result in infertility. Several genes implicated in sexual determination and differentiation have been reported $[3,4,5]$. Gametogenesis, including oogenesis and spermatogenesis, are also regulated by complex mechanisms and several regulatory genes $[6,7]$. A previous study explored regulation of early stages of oogenesis [8]. In addition, studies explored biological mechanisms that occur mid-oogenesis [9], and regulation of late oogenesis [10]. Spermatogenesis is divided into three steps: spermatogonial mitotic proliferation, two times of meiosis, and post-meiotic differentiation [11]. Previous studies explored the functional mechanisms of spermatocytogenesis [12], meiosis during spermatogenesis [13], and spermiogenesis [14].

Fertility of polyploids has important implications in fisheries and sustainable aquaculture. Artificial triploids of species such as Atlantic salmon, Oncorhynchus mykiss, Salmo trutta and Salvelinus fontinalis have been widely used in fish farming industry [15]. There has been a believe that triploidization causes infertility in fish. In addition, a previous study reports that the triploid can channel the energy required for gonad maturation to somatic growth, causing rapid growth rates compared with their diploid counterparts [16]. However, a different study reported that triploid fish can produce normal gametes [17]. In our previous study, Carassius auratus red var. (RCC) (female) and autotetraploid Carassius auratus (4nRR) (male) were artificially hybridized to produce hybrid autotriploid Carassius auratus (3nRR). After hybridization, the male $3 \mathrm{nRR}$ did not produce normal sperm, whereas the females generated dynamic eggs [18]. Analysis of meiosis-related gene expression showed that $D m c 1$ and $P h 1$ had higher expression level in female 3nRR compared with levels in the males, indicating that these genes are involved in regulating fertility of $3 n R R$ [19]. Molecular mechanisms involving 3nRR fish in controlling fertility have not been explored fully.

RNA-Seq technology is utilised for analysis of the structure and function of genes at the organismal level, and for exploring a series of biological pathways [20]. RNA-Seq technique has been successfully used in studies various fishes in the past decade. In spotted scat species (Scatophagus argus), several candidate genes involved in reproduction and gonadal development were obtained by RNA-Seq [21]. Studies on Takifugu rubripes reported that sex-related genes play an important role at early sex differentiation stage [22]. Gonadal transcriptome profiling of triploid hybrid loaches (Misgurnus anguillicaudatus) and their diploid and tetraploid parents showed key genes implicated in low hybrid triploid fertility [17]. A study on Thunnus maccoyii reported sex and gonad-development-related genes in the gonads of Southern bluefin tuna through RNA-Seq [23]. In addition, RNA-Seq has been successfully used to analyze sex determination and differentiation related genes in tilapia [24]. In the present study, we successfully obtained triploid fish ( $3 n R R)$ by crossing female RCC and male $4 n R R$. The diploid $\left(2 \mathrm{nF}_{1}\right)$, triploid $\left(3 \mathrm{nF}_{1}\right)$ and tetraploid $\left(4 \mathrm{nF}_{1}\right)$ hybrids were then generated by hybridization of female $3 \mathrm{nRR}$ and male RCC. In this study, we explored important biological traits and systematically compared gonadal transcriptome of the triploid fish (3nRR) with their parents. Further, the molecular mechanism of the low fertility of the 
autotriploid fish was explored. The findings of this study provide information on the biological characteristics of 3nRR and mechanisms associated with fertility regulation in triploid fish.

\section{Results}

\section{Generation of autotriploid Carassius auratus}

3nRR were generated by crossing female RCC and male 4nRR during the breeding season (Fig. 1a, b, c, Fig. 2). Chromosome number analysis showed that $92.5 \%$ of metaphases had 100 chromosomes in RCC, implying that they were diploids with 100 chromosomes $(2 n=100)$ (Fig. 3a, Table 1$)$. Analysis of $4 n R R$ showed that $87.0 \%$ of metaphases had 200 chromosomes, implying that they were tetraploids with 200 chromosomes $(4 n=200)($ Fig. 3b, Table 1$)$. Analysis of $3 n R R$ showed that $91.5 \%$ of metaphases had 150 chromosomes, suggesting that they were triploids with 150 chromosomes $(3 n=150)($ Fig. 3c, Table 1$)$.

\section{Fertility of autotriploid Carassius auratus}

Testes of RCC and 4nRR (Fig. 4a, b) contained spermatogonia (SG), spermatocytes (SC) and a large number of mature spermatid (ST), whereas the mature sperm was not observed in 3nRR (Fig. 4c). Ovaries of RCC, 4nRR and 3nRR contained second, third and fourth phase oocytes (Fig. 4d, e, f). These results indicated that all ovaries, and RCC and 4nRR testes were fertile whereas $3 \mathrm{nRR}$ testes were sterile.

Different sizes of eggs and water-like semen were collected during the reproductive season from two years old males and females of $3 \mathrm{nRR}$, respectively (Fig. 5). Ploidy levels of the offspring resulting from a cross of female $3 n R R$ and male RCC (Figure 1c, d, e, f, g) were determined by measuring the chromosome number (Figure 3d, e, f, Table 1). Analysis showed that female 3nRR produced haploid, diploid and triploid eggs.

\section{Transcriptome sequencing and sequence alignment}

Optical density (OD) ratio A260/A280 and RNA integrity numbers (RINs) of the RNA in 12 samples (Table 2) were 2.1 and 8.0-8.8, respectively (Additional file 1). These results indicate that all samples were free from contamination and their quality met the requirements for transcriptome sequencing.

RNA-seq from gonadal tissue samples of autotriploid fish and their parents was performed by Illumina. RNA-Seq results are presented in Table 3 and Table 4. Number of clean reads from the 12 RNA-seq libraries ranged from $39,624,312$ to $50,588,484$. All clean reads were then aligned to the RCC genome sequences using HISAT2 software. Mapped genome reads ranged from 24,237,536 to 42,474,296, genome map rates ranged from $59.79 \%$ to $91.55 \%$, and unique match rates ranged from $57.84 \%$ to $85.93 \%$.

\section{Identification of differentially expressed genes (DEGs)}


Analysis of F3nRR and FRCC showed that a total of 13,467 DEGs were downregulated whereas 6,688 DEGs were up-regulated (Fig. 6a). DEGs between F3nRR and FRCC included forkhead box L2 (FOXL2), LIM homeobox 8 (LHX8), lysine acetyltransferase 8 (KAT8), BCL2 apoptosis regulator (BCL2), doublesex and mab-3 related transcription factor 1 (DMRT1), ovarian serine protease (OSP) and CCM2 scaffold protein (CCM2). Analysis of M3nRR and F4nRR showed that a total of 1,886 DEGs were downregulated and 2,221 DEGs were up-regulated (Fig. 6b). DEGs between M3nRR and F4nRR included septin 12 (SEPT12), ATPase copper transporting beta (ATP7B), CF transmembrane conductance regulator (CFTR), cAMP responsive element modulator (CREM), cytochrome P450 family 26 subfamily B member 1 (CYP26B1), EF-hand calcium binding domain 2 (EFCAB2) and inhibitor of kappa light polypeptide gene enhancer in B-cells and kinase complex-associated protein (IKBKAP).

\section{GO and KEGG enrichment analysis of DEGs}

$\mathrm{GO}$ enrichment analysis of the biological process, cellular component and molecular function categories yielded 242, 38 and 51 terms, respectively, for F3nRR vs FRCC, and 223, 28 and 29 for M3nRR vs M4nRR group. (Additional file 2,3). The most-enriched GO-terms for F3nRR vs FRCC group were "induction of programmed cell death" in the biological process category, "neuron projection" in the cellular component category, and "channel activity" and "passive transmembrane transporter activity" in the molecular function category. The most-enriched G0-terms for M3nRR vs F4nRR group were "extracellular region part" in the cellular component category; "kinase activity" and "transferase activity, transferring phosphorus-containing groups" in the molecular function category; and "response to osmotic stress" in the biological process category (Fig. 7).

KEGG analysis of all DEGs showed that 44 and 62 signaling pathways were enriched in the F3nRR vs FRCC group and M3nRR vs M4nRR group, respectively (Additional file 4, 5). The top 20 most enriched KEGG pathways are shown in Figure 8. The five most-enriched pathways in the F3nRR vs FRCC group were "ion channels" (ko04040), "cAMP signaling pathway" (ko04024), "focal adhesion" (ko04510), "glycosaminoglycan binding proteins" (ko00536) and "glycosyltransferases" (ko01003). Moreover, several pathways implicated in female fertility of F3nRR were identified, including "MAPK signaling pathway - plant" (ko04016), and "p53 signaling pathway" (ko04115). The five most enriched pathways for the M3nRR vs M4nRR group were "Ion channels" (ko04040), "rap1 signaling pathway" (ko04015), "ras signaling pathway" (ko04014), "alcoholism" (ko05034) and "axon guidance" (ko04360). Notably, four of the top 20 most-enriched pathways, "regulation of actin cytoskeleton" (ko04810), "calcium signaling pathway" (ko04020), "tight junction" (ko04530) and "cytokines and growth factors" (ko04052), play important roles in cellular processes such as differentiation, proliferation, migration and apoptosis, implying that they are potentially involved in male sterility of M3nRR.

\section{Hub genes related to the fertility in $3 n R R$ were identified}

GO and KEGG enrichment analyses and published literature showed that a total of 80 genes out of the DEGs identified in the F3nRR vs FRCC group were female fertility-related genes. On the other hand, 25 genes out of the DEGs in the M3nRR vs M4nRR group are implicated in male sterility. To further identify 
hub genes associated with 3nRR fertility, PPI of the fertility-related genes was constructed using STRING tool and analysis was carried out using Cytoscape software. Analysis of PPI network of female fertilityrelated genes showed that 6 hub genes with degrees more than 15 showed strong interaction with other node proteins (Fig. 9a, Additional file 6). Furthermore, PPI of male sterility-related genes showed that 2 hub genes, with degrees more than 5 showed strong interaction with other node proteins (Fig. 9b, Additional file 7).

\section{RT-qPCR verification of the DEGs}

To verify RNA-Seq results, twenty DEGs were chosen for validation by RT-qPCR. Among the 20 DEGs, 5 DEGs were up-regulated in the F3nRR vs FRCC and M3nRR vs M4nRR groups, respectively; whereas 5 DEGs were down-regulated in the F3nRR vs FRCC and M3nRR vs M4nRR groups, respectively. Expression profiles of the twenty DEGs obtained by RT-qPCR and RNA-Seq were similar, implying that RNA-Seq results were reliable (Fig. 10).

\section{Discussion}

Triploid animals are usually sterile and cannot form triploid populations. However, previous studies have been reporting contradicting results. Xiao et al. [25] reported that triploid Carassius auratus in Dongting water system produces normal gametes. Hu et al. [26] reported that female autotriploid hybrids (3nAUT) generated by crossing females of Carassius auratus red var. with males of autotetraploid fish produced mature eggs. However, male 3nAUT showed abnormal gonadal development and could not produce mature sperm. In the present study, 3nRR was generated by crossing female RCC and male 4nRR. Three different ploidy offspring were then obtained by hybridization of female 3nRR and male RCC. The female 3nRR offspring were fertile, whereas male 3nRR were sterile. However, only a few studies have explored the molecular mechanisms modulating fertility of the autotriploid of Carassius auratus. In this study transcriptome analysis was used to explore the molecular mechanisms associated with poor fertility in 3nRR. Eight fertility-related hub genes of 3nRR were identified through GO and KEGG enrichment analyses, and previous published literature.

\section{Candidate hub genes related to male sterility of 3nRR were identified}

Hub genes identified in the M3nRR vs M4nRR group included several genes involved in male sterility, such as the tumor protein p53 (TP53) and fibroblast growth factor 2 (FGF2).

TP53, also known as $P 53$ is a transcriptional regulator and tumor suppressor implicated in spermatogenesis [27]. In vertebrates, partial or complete impairment of $P 53$ expression causes disordered meiotic divisions, which in turn causes spermatogenesis defects $[28,29]$. P53 mRNA and protein levels are downregulated in the testis of $P 53$ promoter-chloramphenicol acetyltransferase (CAT)-harboring mice, indicating its important role in development of spermatocytes [30]. In addition, TP53 codon 72 polymorphism in mice is involved in meiosis, implying that it plays a critical role in spermatogenesis [31]. In human, P53 gene polymorphism is higher in infertile men compared with fertile men, implying that it 
may affect germ cell apoptosis and increase risk of male infertility [29,32]. In the current study, analysis of expression levels of the TP53 gene showed significantly different expression levels between M3nRR and M4nRR. This finding implies that TP53 may disrupt meiosis during spermatogenesis in the male 3nRR fish causing sterility.

Fibroblast growth factor 2 (FGF2) plays essential functions in regulation of spermatogenesis and sperm physiology [33]. A study using a human model reported presence of FGF2 and FGFRs in testis and sperm, which are related with human spermatogenesis and sperm motility [34]. Furthermore, incubation of human sperm with recombinant $F G F 2$ ( $\mathrm{r} F G F 2$ ) causes an increase in number of motile cells, implying that the gene is involved in sperm motility [35]. In mouse, knock out of FGF2 induces impaired sperm production and is associated with alterations in sperm morphology and function [36]. In this study, FGF2 was significantly downregulated in M3nRR vs 4nRR. Low expression levels of the gene can cause abnormal shaping of the normal sperm, which resulted in male 3nRR sterility.

\section{Candidate hub genes related to female fertility of 3nRR were identified}

Six hub genes associated with female fertility were identified in the F3nRR vs F4nRR group including MYC proto-oncogene, bHLH transcription factor $(M Y C)$, SRY-box transcription factor 2 (SOX2), bone morphogenetic protein 4 (BMP4), GATA binding protein 4 (GATA4), phosphatase and tensin homolog (PTEN) and bone morphogenetic protein 2 (BMP2).

MYC gene encodes the MYC transcription factor which is involved in cell proliferation and gametogenesis [37]. In Xenopus, C-MYC was detected in oocytes, indicating that it plays a role in oogenesis [38]. In Drosophila, MYC was involved in distribution of CTPsyn in follicle cells, implying that it plays a role in synthesizing nutrients for the developing oocytes [39]. In addition, a previous study reported that MYC plays an important role in regulation of mitochondrial biogenesis in Drosophila ovary, indicating that the gene is involved in oocyte development [40]. In Larimichthys crocea, Lc-cMYC had different expression patterns in oocytes at various stages of development, implying that it plays an essential role in oogenesis [41]. In the current study, 3nRR ovary showed low expression level of $M Y C$ which may inhibited formation of oogenesis defects during triploidization, resulting in production mature eggs.

SOX is an ancient gene family involved in oogenesis [42]. Sox genes have been explored in many organisms and can be classified into ten subgroups (A-J) [43]. In Agasicles hygrophila, AhDichaete and AhSox 3 expression levels are significantly high in ovary, indicating that it plays a vital regulatory role in during ovarian development and oogenesis [44]. In Misgurnus anguillicaudatus, MaSOX3 is abundant in primary oocytes and previtellogenic oocyte cells, indicating that MaSox3 gene is involved in ovarian development [45]. In Paramisgurnus dabryanus, SOX4 was detected in the ovary, showing that it plays an important role during ovarian development [46]. In mouse, expression of SOX2 is required for establishment and maintenance of the oocyte cell [47]. In our study, SOX2 was identified in F3nRR vs FRCC, with lower expression level in F3nRR gonad compared with the expression level in FRCC gonad. This finding shows that SOX2 may be an important factor in normal ovarian development of F3nRR. 
Bone morphogenetic proteins (BMPs) are belonging to the transforming growth factor- $\beta$ superfamily of proteins, and they appear to be highly conserved [48]. A previous study reports that BMPs play a role in regulation of ovarian follicular development [49]. BMP1, BMP6 and BMP15 are implicated in ovarian development [50, 51, 52]. In Xenopus laevis, $B M P 2$ gene is highly expressed during oogenesis, implying that it is an important factor in ovarian development [53]. In mouse, BMP4 regulates the number of oocytes, suggesting its role in the process of oogenesis [54]. Roles of BMP2 and BMP4 as important factors in survival and development of bovine secondary follicles were recently reported [55]. In this study, $B M P 2$ and $B M P 4$ genes were differentially expressed between F3nRR and FRCC, indicating that they may be involved in ovarian development.

GATA4, a member of the GATA-binding family, is highly expressed in ovarian granulosa cells [56, 57]. A previous study reported that the gene is involved in regulation of ovarian development [58]. GATA4 and GATA6 knockout female mice exhibited infertility due to disrupted formation of ovaries [59]. GATA4 deletion resulted in a sterile female mice phenotype attributed to drastic reduction in number of developing follicles [60]. The mRNA for GATA4 has been reported in human ovary implying that GATA4 plays a role in ovarian folliculogenesis [57]. GATA4 was identified through transcriptome analysis in this study. GATA4 was differentially expressed in F3nRR vs FRCC, indicating that plays important roles in ovarian development of 3nRR.

Phosphatase and tensin homolog (PTEN) protein has phosphatase activity and belongs to proteintyrosine phosphatase superfamily [61]. PTEN is a negative regulator of PI3K-Akt signaling pathway which is involved in growth of eggs [62]. Deletion of PTEN from oocytes affects mouse fertility by interrupting oocyte growth [63]. PTEN signaling pathway associated with ovarian follicle development has been reported in human [64]. In Crassostrea gigas, PTEN is involved in insulin pathway in gonads and plays a critical role in reproduction [65]. In Drosophila, loss of PTEN is related to IIS/mTORC1 signalling, which is important for oogenesis [66]. In the present study, F3nRR showed significantly low expression levels of PTEN, which may have caused F3nRR normal fertility through regulation of oogenesis.

\section{Conclusions}

The autotriploid Carassius auratus ( $3 n R R, 3 n=150)$ is generated from Carassius auratus red var. (RCC, $2 n=100)(\mathbb{Z})$ and autotetraploid Carassius auratus $(4 n R R, 4 n=200)(\mathbb{Z})$, of which the female $3 n R R$ can produce mature gametes, whereas the male $3 n R R$ cannot. In addition, we produced diploid $\left(2 n F_{1}, 2 n=\right.$ $100)$, triploid $\left(3 n F_{1}, 3 n=150\right)$ and tetraploid $\left(4 n F_{1}, 4 n=200\right)$ hybrids in the $F_{1}$ generation by crossing females of 3nRR with males of RCC, which further indicated that female 3nRR were fertile. Gonadal transcriptome reveals 6 hub genes (MYC, SOX2, BMP4, GATA4, PTEN and BMP2) were involved in the female fertility of the female $3 \mathrm{nRR}$, and 2 hub genes ( $T P 53$ and FGF2) were involved in the male sterility of the male $3 n R R$. The obtained data reveals novel candidate genes for the fertility in the autotriploid fish and also extends an understanding of the molecular aspects of fertility in triploid fish.

\section{Methods}




\section{Animals and crosses}

One year old RCC and one year old 4nRR $\left(F_{11}\right)$ were fed in the State Key Laboratory of Developmental Biology of Freshwater Fish, Hunan Normal University, China. Hybrids (3nRR) of RCC ( ()$\times 4 n R R(\nabla)$ were generated in May 2018. All fish were maintained in open pools (0.067 ha) with suitable pH (7.0-8.5), water temperature $\left(22-24^{\circ} \mathrm{C}\right)$, dissolved oxygen content $(5.0-8.0 \mathrm{mg} / \mathrm{L})$ and adequate forage. All dissections were performed under MS-222 anaesthesia (100 mg/L; Sigma-Aldrich).

\section{Gonadal histologic analysis}

Ploidy levels of the fish (RCC, 4nRR and 3nRR) were estimated using a flow cytometer (Gallios Flow Cytometer, Beckman Coulter). Blood was collected from the caudal vein using heparinized syringes. Samples were then resuspended in 4,6-Diamidino-2-Phenylindole solution (Sigma-Aldrich) for $10 \mathrm{~min}$. DNA content was compared with that of RCC per sample. Gonadal tissues of two years old female RCC, male 4nRR and 3nRR were fixed in Bonn's liquid and then dehydrated using graded series of alcohol, cleared with xylene, embedded in paraffin wax and cut into 5-8 $\mu \mathrm{m}$ sections. The sections were placed on slides, stained with hematoxylin and eosin, and viewed under a light microscope.

\section{Gamete phenotypes and egg ploidy detection}

The water-like semen and mature eggs of two years old 3nRR were sampled for morphological examination. The female 3nRR produced different sized eggs. To determine the egg ploidy, mature eggs were used in vitro fertilization of the RCC haploid sperm and then viable offspring $\left(2 n F_{1}, 3 n F_{1}\right.$ and $\left.4 \mathrm{nF}_{1}\right)$ were generated. Ploidy of these offspring was detected by chromosome counts.

\section{Preparation of chromosome spreads}

For ploidy level analysis, chromosome counts were carried out using kidney tissues from 10 individuals each of RCC, $4 n R R, 3 n R R, 2 n F_{1}, 3 n F_{1}$ and $4 \mathrm{nF}_{1}$ at eight months of age following a previously described method [67]. 200 metaphase chromosome spreads (20 spreads per sample) were analyzed for each type of fish. Each preparation was examined under 3330× magnification with an oil immersion lens.

\section{Sample collection and preparation for transcriptomic sequencing}

A total of 3 females RCC (FRCC), 3 males 4nRR (M4nRR), 3 males 3nRR (M3nRR) and 3 females 3nRR (F3nRR) were acquired at 24 months. Fish were anesthetized before surgical removal of tissues. Gonadal tissues were harvested from FRCC, M4nRR, F3nRR and M3nRR after euthanasia.

\section{RNA extraction and sequencing}

Total RNA was extracted from gonads of RCC, 4nRR and 3nRR using TRIzol reagent (Takara, Beijing, China) according to the instructions. RNA integrity (RNA integrity score $\geq 7.0$ ) was checked on the bioanalyzer 2100 system (Agilent, Palo Alto, CA) and RNA quantity was measured using NanoDrop 2000 
(Thermo, Waltham, MA, USA). A total of 12 libraries from FRCC, M4nRR, F3nRR and M3nRR groups were sequenced. In summary, mRNA was purified and broken into short fragments. Then, reverse transcription, cDNA synthesis and cluster generation were performed. RNA-Seq libraries were then sequenced on Illumina Hiseq2500 platform. The sequenced data are publicly available at the NCBI (PRJNA694292).

\section{Differential expressed genes (DEGs) and profiling of potential fertility-related genes}

After sequencing, clean reads were acquired by removing adapters and low-quality reads using fastp software (Version 0.20.0). The clean reads quality was assessed with FastQC software (Version 0.11.9). The clean reads of the libraries were aligned to the published RCC reference genome (https://bigd.big.ac.cn/search?dbld=gwh\&q=GWHAAIA00000000) using HISAT2 tool (Version 2.1.0). To calculate gene expression level, we used fragments per kilobase per million mapped fragments (FPKM) method. Differentially expressed genes (DEGs) analysis of F3nRR vs FRCC, and M3nRR vs M4nRR was performed using DEGSeq2 R package (Version 1.28.1). Genes having a fold change (FC) $>2$ and a false discovery rate $(F D R)<0.05$ were considered as DEGs. To further explore these DEGs, Gene ontology (GO) and Kyoto Encyclopedia of Genes and Genomes (KEGG) enrichment analysis were performed using clusterProfiler (Version 3.6.0) with $p<0.05$. Moreover, fertility-related DEGs were screened following GO, KEGG enrichment analyses and published literature. STRING database (https://string-db.org/) to construct protein-protein interaction (PPI) networks to explore protein relationships among the fertilityrelated DEGs. Hub genes were obtained based on the ranking order of connectivity degree by Cytoscape software [68].

\section{Quantitative Real-time PCR verification}

Ten significantly DEGs (five up-regulated DEGs and five down-regulated DEGs) were chosen for quantitative real-time (qRT) PCR to test the reliability of the F3nRR vs FRCC and M3nRR vs M4nRR transcriptome sequencing results. PrimeScript ${ }^{\mathrm{TM}} \mathrm{RT}$ reagent kit (Takara, Dalian, China) was used to perform cDNA synthesis following the manufacturer's instructions. Primer sequences for $\beta$-actin (the internal control gene) and these DEGs are listed in Additional file 8. The 10- $\mu$ l-volume qRT-PCR reaction mixture consisted of $5 \mu \mathrm{l}$ SYBR Green qPCR Master Mix, $0.5 \mu \mathrm{l}$ of $20 \mu \mathrm{M}$ of each primer, $1 \mu \mathrm{l}$ of cDNA (1:10 dilution) and $3 \mu \mathrm{L}$ of nuclease-free water. qRT-PCR thermal cycle used was as follows: $95^{\circ} \mathrm{C}$ for 2 min, 40 cycles of $95^{\circ} \mathrm{C}$ for $15 \mathrm{~s}$ and annealing at $60^{\circ} \mathrm{C}$ for $30 \mathrm{~s}$. Three technical replicates were used for each biological sample in the qRT-PCR. Relative mRNA expression level was calculated by using the $2^{-\triangle \Delta C t}$ method. Data were analyzed statistically using SPSS (v22.0) software (SPSS Inc., Chicago, IL, USA). Statistical significance was determined using Student's t-test analysis.

\section{Abbreviations}

RCC

Carassius auratus red var.;

3nRR 
autotriploid Carassius auratus;

4nRR

autotetraploid Carassius auratus;

FRCC

female Carassius auratus red var.;

M4nRR

male autotetraploid Carassius auratus,

F3nRR

female autotriploid Carassius auratus;

M3nRR

male autotriploid Carassius auratus;

PPI

protein-protein interaction;

$2 \mathrm{nF}_{1}$

diploid hybrids of female autotriploid Carassius auratus $\times$ male Carassius auratus red var.; $3 \mathrm{nF}_{1}$

triploid hybrids of female autotriploid Carassius auratus $\times$ male Carassius auratus red var.; $4 \mathrm{nF}_{1}$

tetraploid hybrids of female autotriploid Carassius auratus $\times$ male Carassius auratus red var.;

SG

spermatogonia;

$\mathrm{SC}$

spermatocytes;

ST

spermatid;

DEGs

differentially expressed genes;

GO

Gene Ontology;

KEGG

Kyoto Encyclopedia of Genes and Genomes;

TP53

tumor protein $\mathrm{p} 53$;

FGF2

fibroblast growth factor 2;

MYC

MYC proto-oncogene, bHLH transcription factor;

SOX2

SRY-box transcription factor 2;

BMP4 
bone morphogenetic protein 4;

GATA4

GATA binding protein 4;

PTEN

phosphatase and tensin homolog;

$B M P 2$

bone morphogenetic protein 2 .

\section{Declarations}

\section{Ethics approval and consent to participate}

The study was approved by Ethics Committee of Hunan Normal University, all methods were carried out in accordance with relevant guidelines and regulations. This study was carried out in compliance with the ARRIVE guidelines.

\section{Consent for publication}

Not applicable.

\section{Availability of data and materials}

Raw sequence reads are available from the NCBI (PRJNA694292)

(https://www.ncbi.nlm.nih.gov/bioproject/PRJNA694292).

\section{Competing interests}

The authors declare that there are no competing financial interests.

\section{Funding}

This work was supported by the Natural Science Foundation for Distinguished Young Scholars of Hunan Province (Grant No. 2017JJ1022), the National Natural Science Foundation of China (Grant No. 31730098, U19A2040), the Earmarked Fund for China Agriculture Research System (Grant No. CARS-45), Hunan Provincial Science and Technology Department (2019RS5001) and the National Key Research and Development Program of China (2020YFD0900104).

\section{Authors' contributions}

SL and QQ have designed of the work. CW has contributed to this study for the design, in executing experiments and in writing manuscript. $X L, H Q$ and $C Z$ have made substantial contributions to the acquisition and analysis of data. $\mathrm{LY}, \mathrm{TY}, \mathrm{YZ}, \mathrm{XH}$ and $\mathrm{XX}$ have substantively revised the work.

\section{Acknowledgements}


Not applicable.

\section{References}

1. Otto SP, Whitton J. Polyploid incidence and evolution. Annu Rev Genet. 2000; 34(1), 401-37.

2. Phizicky DV, Berchowitz LE, Bell SP. Multiple kinases inhibit origin licensing and helicase activation to ensure reductive cell division during meiosis. Elife. 2018; 7: e33309.

3. Nagahama Y. Molecular mechanisms of sex determination and gonadal sex differentiation in fish. Fish Physiol Biochem. 2005; 31(2-3): 105-9.

4. Quinn A, Koopman P. The molecular genetics of sex determination and sex reversal in mammals. Semin Reprod Med. 2012; 30(5): 351-63.

5. Windley SP, Wilhelm D. Signaling pathways involved in mammalian sex determination and gonad development. Sex Dev. 2015; 9(6): 297-315.

6. Schultz N, Hamra FK, Garbers DL. A multitude of genes expressed solely in meiotic or postmeiotic spermatogenic cells offers a myriad of contraceptive targets. Proc Natl Acad Sci. 2003; 100: 122016.

7. Lee CS, Lu T, Seydoux G. Nanos promotes epigenetic reprograming of the germline by downregulation of the thap transcription factor lin-15b. Elife. 2017; 6: e30201.

8. Marco N, Assa Y, Eckmann CR. Stage-specific combinations of opposing poly(a) modifying enzymes guide gene expression during early oogenesis. Nucleic Acids Research. 2019; 20: 20.

9. Kong J, Han H, Bergalet J, Bouvrette LPB, Hernández G, Moon NS, et al. A ribosomal protein S5 isoform is essential for oogenesis and interacts with distinct RNAs in Drosophila melanogaster. Scientific Reports. 2019; 9(1): 1-11.

10. Kawaguchi S, Ueki M, Kai T. Drosophila MARF1 ensures proper oocyte maturation by regulating nanos expression. PLoS One. 2020; 15(4): e0231114.

11. Snyder E, Chukrallah L, Seltzer K, Goodwin L, Braun RE. ADAD1 and ADAD2, testis-specific adenosine deaminase domain-containing proteins, are required for male fertility. Scientific Reports. 2020; 10(1).

12. Phillips BT, Gassei K, Orwig KE. Spermatogonial stem cell regulation and spermatogenesis. Phil. Trans. R. Soc. B. 2010; 365: 1663-78.

13. Wolgemuth DJ, Roberts SS. Regulating mitosis and meiosis in the male germ line: critical functions for cyclins. Phil. Trans. R. Soc. B. 2010; 365: 1653-62.

14. Bettegowda A, Wilkinson MF. Transcription and post-transcriptional regulation of spermatogenesis. Phil. Trans. R. Soc. B. 2010; 365: 1637-51.

15. Piferrer F, Beaumont A, Falguiere JC, Flajshans M, Haffray P, Colombo L. Polyploid fish and shellfish: production, biology and applications to aquaculture for performance improvement and genetic containment. Aquaculture. 2009; 293: 125-56.

16. Cal RM, Vidal S, Gómez C, Álvarez-Blázquez B, Martínez P, Piferrer F. Growth and gonadal development in diploid and triploid turbot (Scophthalmus maximus). Aquaculture. 2006; 251(1): 99 - 
108.

17. He Z, Xu QZ, Rui Z, Zhuang ZX, Ma YQ, Wei W, et al. Gonadal transcriptome analysis of hybrid triploid loaches (Misgurnus anguillicaudatus) and their diploid and tetraploid parents. PLoS One. 2018; 13(5): e0198179.

18. Qin QB, Wang YD, Wang J, Dai J, Xiao J, Hu FZ, et al. The autotetraploid fish derived from hybridization of Carassius auratus red var. (female) $\times$ Megalobrama amblycephala (male). Biol Reprod. 2014; 91(4): 93.

19. Qin QB, Zhou YW, Wang CQ, Zhang MH, Qin H, Zhao C, et al. Analysis on the meiosis-related gene (Dmc1, Ph1) expression in autotriploid Carassius auratus. Mar Biotechnol. 2019; 21: 753-61.

20. Qian X, Ba Y, Zhuang QF, Zhong GF. RNA-Seq technology and its application in fish transcriptomics. OMICS. 2014; 18(2): 98-110.

21. Yao Q, Dong YZ, Chen J, Quan LF, Zhang WQ, Chen BX. Transcriptome analysis of female and male Conopomorpha sinensis (Lepidoptera: Gracilariidae) adults with a focus on hormone and reproduction. J Econ Entomol. 2019; 112(6): 2966-75.

22. Yan HW, Shen XF, Cui X, Wu YW, Wang LS, Zhang L, et al. Identification of genes involved in gonadal sex differentiation and the dimorphic expression pattern in Takifugu rubripes gonad at the early stage of sex differentiation. Fish Physiol Biochem. 2018; 44(5): 1275-90.

23. Bar I, Cummins S, Elizur A. Transcriptome analysis reveals differentially expressed genes associated with germ cell and gonad development in the Southern bluefin tuna (Thunnus maccoyii). BMC Genomics. 2016; 17: 217.

24. Tao W.J, Chen JL, Tan DJ, Yang J, Sun L, Wei J, et al. Transcriptome display during tilapia sex determination and differentiation as revealed by RNA-Seq analysis. BMC Genomics. 2018; 19(1): 363.

25. Xiao J, Zou TM, Chen YB, Chen L, Liu SJ, Tao M, et al. Coexistence of diploid, triploid and tetraploid crucian carp (Carassius auratus) in natural waters. BMC Genet. 2011; 12(1), 20.

26. Hu F, Fan JJ, Qin QB, Huo YY, Wang YD, Wu C, et al. The sterility of allotriploid fish and fertility of female autotriploid fish. Front. Genet. 2019; 10: 377.

27. Almon E, Goldfinger N, Kapon A, Schwartz D, Levine AJ, Rotter V. Testicular tissue-specific expression of the p53 suppressor gene. Dev Biol. 1993: 156(1): 107-16.

28. Harvey M, McArthur MJ, Montgomery CA, Bradley A, Donehower LA. Genetic background alters the spectrum of tumors that develop in p53-deficient mice. FASEB J. 1993; 7: 938-43.

29. Huang C, Liu W, Ji GX, Gu AH, Qu JH, Song L, et al. Genetic variants in TP53 and MDM2 associated with male infertility in Chinese population. Asian J Androl. 2012; 14(5): 691-4.

30. Rotter V, Schwartz D, Almon E, Goldfinger N, Kapon A, Meshorer A, et al. Mice with reduced levels of p53 protein exhibit the testicular giant-cell degenerative syndrome. PProc Natl Acad Sci U S A. 1993; 90(19): 9075-9. 
31. de Morais MP, Curado RF, Silva KS., Moura K, Arruda JT. Male idiopathic infertility and the TP53 polymorphism in codon 72. Genet Mol Res. 2016; 15(4).

32. Ebrahim Abadi Z, Khademi Bami M, Golzadeh M, Kalantar SM, Sheikhha MH. The frequency of TP53 R72P and MDM2 309T >G polymorphisms in Iranian infertile men with spermatogenetic failure: A case-control study. Int J Reprod Biomed. 2018; 16(8): 491-6.

33. Saucedo L, Rumpel R, Sobarzo C, Schreiner D, Brandes G, Lustig L, et al. Deficiency of fibroblast growth factor 2 (FGF-2) leads to abnormal spermatogenesis and altered sperm physiology. J Cell Physiol. 2018; 233(12), 9640-51.

34. Garbarino Azúa DJ, Saucedo L, Giordana S, Magri ML, Buffone MG, Neuspiller F, et al. Fibroblast growth factor 2 (FGF2) is present in human spermatozoa and is related with sperm motility. The use of recombinant FGF2 to improve motile sperm recovery. Andrology. 2017; 5(5): 990-8.

35. Saucedo L, Buffa GN, Rosso M, Guillardoy T, Góngora A, Munuce MJ, et al. Fibroblast growth factor receptors (FGFRs) in human dperm: expression, functionality and involvement in motility regulation. PLoS One. 2015; 10(5): e0127297.

36. Saucedo L, Sobarzo C, Brukman NG, Guidobaldi HA, Lustig L, Giojalas LC, et al. Involvement of fibroblast growth factor 2 (FGF2) and its receptors in the regulation of mouse sperm physiology. Reproduction. 2018; 156(2): 163-72.

37. Hartl M, Glasauer S, Valovka T, Breuker K, Hobmayer B, Bister K. Hydra myc2, a unique pre-bilaterian member of the myc gene family, is activated in cell proliferation and gametogenesis. Biol Open. 2014; 3(5): 397-407.

38. Godeau F, Persson H, Gray HE, Pardee AB. C-myc expression is dissociated from DNA synthesis and cell division in Xenopus oocyte and early embryonic development. EMBO J. 1986; 5(13): 3571-7.

39. Aughey GN, Grice SJ, Liu JL. The Interplay between Myc and CTP Synthase in Drosophila. PLoS Genet. 2016; 12(2): e1005867.

40. Wang ZH, Liu Y, Chaitankar V, Pirooznia M, Xu H. Electron transport chain biogenesis activated by a JNK-insulin-Myc relay primes mitochondrial inheritance in Drosophila. Elife. 2019; 8: e49309.

41. Jiang Y, Han K, Chen S, Wang Y, Zhang Z. Isolation, characterization, and expression of protooncogene cMyc in large yellow croaker Larimichthys crocea. Fish Physiol Biochem. 2017; 43(5): 1443-61.

42. Phochanukul N, Russell S. No backbone but lots of Sox: Invertebrate Sox genes. Int J Biochem Cell Biol. 2010; 42(3): 453-64.

43. Bowles J, Schepers G, Koopman P. Phylogeny of the SOX family of developmental transcription factors based on sequence and structural indicators. Dev Biol. 2000; 227(2): 239-55.

44. Dong WY, Wang Y, Zhou ZS, Guo JY. Sox genes in Agasicles hygrophila (Coleoptera: Chrysomelidae) are involved in ovarian development and oogenesis. Arch Insect Biochem Physiol. 2020; 105(1): e21721.

45. Xia X, Huo W, Wan R, Zhang L, Xia X, Chang Z. Molecular cloning and expression analysis of Sox3 during gonad and embryonic development in Misgurnus anguillicaudatus. Int J Dev Biol. 2017; 61(8- 
9): 565-70.

46. Xia X, Wan R, Huo W, Zhang L, Xia X, Chang Z. Molecular cloning and mRNA expression pattern of Sox4 in Paramisgurnus dabryanus. Gene Expr Patterns. 2017; 25-26: 109-17.

47. Campolo F, Gori M, Favaro R, Nicolis S, Pellegrini M, Botti F, et al. Essential role of Sox2 for the establishment and maintenance of the germ cell line. Stem Cells. 2013; 31(7): 1408-21.

48. Dumic-Cule I, Peric M, Kucko L, Grgurevic L, Pecina M, Vukicevic S. Bone morphogenetic proteins in fracture repair. Int Orthop. 2018; 42(11): 2619-26.

49. Juengel JL, McNatty KP. The role of proteins of the transforming growth factor-beta superfamily in the intraovarian regulation of follicular development. Hum Reprod Update. 2005; 11(2): 143-60.

50. Peng C, Clelland E, Tan Q. Potential role of bone morphogenetic protein-15 in zebrafish follicle development and oocyte maturation. Comparative biochemistry and physiology. Comp Biochem Physiol A Mol Integr Physiol. 2009; 153(1): 83-7.

51. Lei X, Cui K, Li Z, Su J, Jiang J, Zhang H, et al. BMP-1 participates in the selection and dominance of buffalo follicles by regulating the proliferation and apoptosis of granulosa cells. Theriogenology. 2016; 85(5): 999-1012.

52. Otsuka F. Interaction of melatonin and BMP-6 in ovarian steroidogenesis. Vitam Horm. 2018; 107: 137-53.

53. Clement JH, Fettes P, Knöchel S, Lef J, Knöchel W. Bone morphogenetic protein 2 in the early development of Xenopus laevis. Mech Dev. 1995; 52(2-3): 357-70.

54. Park ES, Woods DC, Tilly JL. Bone morphogenetic protein 4 promotes mammalian oogonial stem cell differentiation via Smad1/5/8 signaling. Fertil Steril. 2013; 100(5): 1468-75.

55. da Cunha EV, Melo L, Sousa GB, Araújo VR, Vasconcelos GL, Silva A, et al. Effect of bone morphogenetic proteins 2 and 4 on survival and development of bovine secondary follicles cultured in vitro. Theriogenology. 2018; 110: 44-51.

56. Heikinheimo M, Ermolaeva M, Bielinska M, Rahman NA, Narita N, Huhtaniemi IT, et al. Expression and hormonal regulation of transcription factors GATA-4 and GATA- 6 in the mouse ovary. Endocrinology. 1997; 138(8): 3505-14.

57. Laitinen MP, Anttonen M, Ketola I, Wilson DB, Ritvos O, Butzow R, et al. Transcription factors GATA-4 and GATA-6 and a GATA family cofactor, FOG-2, are expressed in human ovary and sex cord-derived ovarian tumors. J Clin Endocrinol Metab. 2000; 85(9), 3476-83.

58. Lowry JA, Atchley WR. Molecular evolution of the GATA family of transcription factors: conservation within the DNA-binding domain. J Mol Evol. 2000; 50(2): 103-15.

59. Bennett-Toomey J, Stocco C. GATA regulation and function during the ovarian life cycle. Vitam Horm. 2018; 107: 193-225.

60. Efimenko E, Padua MB, Manuylov NL, Fox SC, Morse DA, Tevosian SG. The transcription factor GATA4 is required for follicular development and normal ovarian function. Dev Biol. 2013; 381(1): 144-58. 
61. Maehama T, Dixon JE. The tumor suppressor, PTEN/MMAC1, dephosphorylates the lipid second messenger, phosphatidylinositol 3,4,5-trisphosphate. J Biol Chem. 1998; 273(22): 13375-8.

62. Adhikari D, Liu K. Molecular mechanisms underlying the activation of mammalian primordial follicles. Endocr Rev. 2009; 30(5): 438-64.

63. Reddy P, Liu L, Adhikari D, Jagarlamudi K, Rajareddy S, Shen Y, et al. Oocyte-specific deletion of Pten causes premature activation of the primordial follicle pool. Science. 2008; 319(5863): 611-3.

64. Ernst EH, Grøndahl M, Grund S, Hardy K, Heuck A, Sunde L, et al. Dormancy and activation of human oocytes from primordial and primary follicles: molecular clues to oocyte regulation. Hum Reprod. 2017; 32(8): 1684-1700.

65. Jouaux A, Franco A, Heude-Berthelin C, Sourdaine P, Blin JL, Mathieu M, et al. Identification of Ras, Pten and p70S6K homologs in the Pacific oyster Crassostrea gigas and diet control of insulin pathway. Gen Comp Endocrinol. 2012; 176(1): 28-38.

66. Mensah LB, Goberdhan D, Wilson C. mTORC1 signalling mediates PI3K-dependent large lipid droplet accumulation in Drosophila ovarian nurse cells. Biol Open. 2017; 6(5), 563-70.

67. Qin QB, Wang J, Wang YD, Liu Y, Liu SJ. Organization and variation analysis of $5 S$ rDNA in

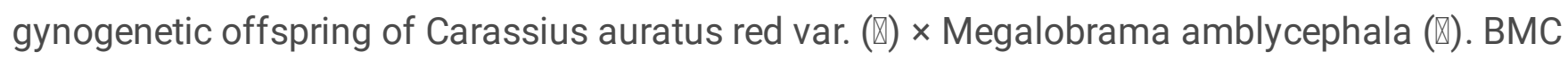
Genetics. 2015; 16: 26.

68. Shannon P, Markiel A, Ozier O, Baliga NS, Wang JT, Ramage D, et al. Cytoscape: A software environment for integrated models of biomolecular interaction networks. Genome Res. 2003; 13 : 2498-504.

\section{Tables}

Table 1 Examination of chromosome number of RCC, $4 n R R, 3 n R R, 2 n F_{1}, 3 n F_{1}$ and $4 n F_{1}$

\begin{tabular}{|llllllll|}
\hline Fish type & No. of & \multicolumn{6}{c|}{ Distribution of chromosome number } \\
\cline { 3 - 8 } & metaphase & $<100$ & 100 & $<150$ & 150 & $<200$ & 200 \\
\hline RCC & 200 & 15 & 185 & & & & \\
$4 n R R$ & 200 & & & & & 26 & 174 \\
\hline $3 n R R$ & 200 & & & 17 & 183 & & \\
\hline $2 n F_{1}$ & 200 & 18 & 182 & & & & \\
$3 n F_{1}$ & 200 & & & 22 & 178 & & \\
$4 \mathrm{nF}_{1}$ & 200 & & & & & 34 & 166 \\
\hline
\end{tabular}

Table 2 Sample information 


\begin{tabular}{|ll|}
\hline Sample No. & Sample type \\
\hline FRCC-1 & RCC-1 (female parent) \\
\hline FRCC-2 & RCC-2 (female parent) \\
\hline M4nRR-3 & RCC-3 (female parent) \\
\hline M4nRR-2 & $4 n R R-1$ (male parent) \\
\hline M4nRR-3 & $4 n R R-2$ (male parent) \\
\hline F3nRR-1 & $F_{1}$ (male) (RCC $\left.\times 4 n R R\right)-1$ \\
\hline F3nRR-2 & $F_{1}$ (male) (RCC $\left.\times 4 n R R\right)-2$ \\
\hline F3nRR-3 & $F_{1}$ (male) (RCC $\left.\times 4 n R R\right)-3$ \\
\hline M3nRR-1 & $F_{1}$ (female) $($ RCC $\times 4 n R R)-1$ \\
\hline M3nRR-2 & $F_{1}$ (female) $($ RCC $\times 4 n R R)-2$ \\
\hline M3nRR-3 & $F_{1}$ (female) $($ RCC $\times 4 n R R)-3$ \\
\hline
\end{tabular}

Table 3 Summary of the RNA-Seq data collected from FRCC, M4nRR, F3nRR and M3nRR 


\begin{tabular}{|lllllll|}
\hline Sample name & Raw reads & Clean reads & Clean bases & Q20 (\%) & Q30 (\%) & GC content (\%) \\
\hline FRCC-1 & $42,334,070$ & $42,252,766$ & $6.31 G$ & 97.83 & 93.94 & 48.99 \\
\hline FRCC-2 & $39,685,218$ & $39,624,312$ & $5.92 G$ & 97.81 & 93.88 & 48.20 \\
\hline FRCC-3 & $46,465,082$ & $46,395,874$ & $6.94 G$ & 97.79 & 93.75 & 48.18 \\
\hline M4nRR-1 & $42,549,848$ & $42,506,792$ & $6.34 G$ & 97.63 & 93.34 & 45.99 \\
\hline M4nRR-2 & $42,757,282$ & $42,709,522$ & $6.36 G$ & 97.65 & 93.46 & 46.84 \\
\hline M4nRR-3 & $40,576,492$ & $40,538,356$ & $6.05 G$ & 97.84 & 93.80 & 45.67 \\
\hline F3nRR-1 & $40,938,734$ & $40,848,106$ & $6.07 G$ & 97.17 & 92.48 & 46.81 \\
\hline F3nRR-2 & $50,703,558$ & $50,588,484$ & $7.54 G$ & 97.43 & 93.03 & 47.14 \\
\hline F3nRR-3 & $48,905,962$ & $48,796,080$ & $7.28 G$ & 97.48 & 93.14 & 47.23 \\
\hline M3nRR-1 & $45,040,604$ & $44,995,054$ & $6.70 G$ & 97.72 & 93.57 & 46.43 \\
\hline M3nRR-2 & $44,702,364$ & $44,659,854$ & $6.67 G$ & 98.09 & 94.25 & 45.66 \\
\hline M3nRR-3 & $45,015,326$ & $44,976,210$ & $6.72 G$ & 97.75 & 93.57 & 45.70 \\
\hline
\end{tabular}

Table 4 Summary of clean reads mapped from FRCC, M4nRR, F3nRR and M3nRR to the reference genome

\begin{tabular}{|lllll|}
\hline Sample name & Total reads & Total mapped & Multiple mapped & Uniquely mapped \\
\hline FRCC-1 & 42252766 & $38,590,536(91.33 \%)$ & $2,470,549(5.85 \%)$ & $36,119,987(85.48 \%)$ \\
\hline FRCC-2 & $39,624,312$ & $36,222,309(91.41 \%)$ & $2,283,305(5.76 \%)$ & $33,939,004(85.65 \%)$ \\
\hline FRCC-3 & $46,395,874$ & $42,474,296(91.55 \%)$ & $2,608,537(5.62 \%)$ & $39,865,759(85.93 \%)$ \\
\hline M4nRR-1 & $42,506,792$ & $25,481,741(59.95 \%)$ & $791,414(1.86 \%)$ & $24,690,327(58.09 \%)$ \\
\hline M4nRR-2 & $42,709,522$ & $26,274,265(61.52 \%)$ & $1,570,067(3.68 \%)$ & $24,704,198(57.84 \%)$ \\
\hline M4nRR-3 & $40,538,356$ & $24,237,536(59.79 \%)$ & $749,604(1.85 \%)$ & $23,487,932(57.94 \%)$ \\
\hline F3nRR-1 & $40,848,106$ & $28,790,170(70.48 \%)$ & $1,218,302(2.98 \%)$ & $27,571,868(67.50 \%)$ \\
\hline F3nRR-2 & $50,588,484$ & $35,839,842(70.85 \%)$ & $1,526,075(3.02 \%)$ & $34,313,767(67.83 \%)$ \\
\hline F3nRR-3 & $48,796,080$ & $34,635,763(70.98 \%)$ & $1,489,678(3.05 \%)$ & $33,146,085(67.93 \%)$ \\
\hline M3nRR-1 & $44,995,054$ & $31,933,903(70.97 \%)$ & $1,010,710(2.25 \%)$ & $30,923,193(68.72 \%)$ \\
\hline M3nRR-2 & $44,659,854$ & $31,782,067(71.16 \%)$ & $982,486(2.20 \%)$ & $30,799,581(68.96 \%)$ \\
\hline M3nRR-3 & $44,976,210$ & $31,860,677(70.84 \%)$ & $1,043,971(2.32 \%)$ & $30,816,706(68.52 \%)$ \\
\hline
\end{tabular}


Figures
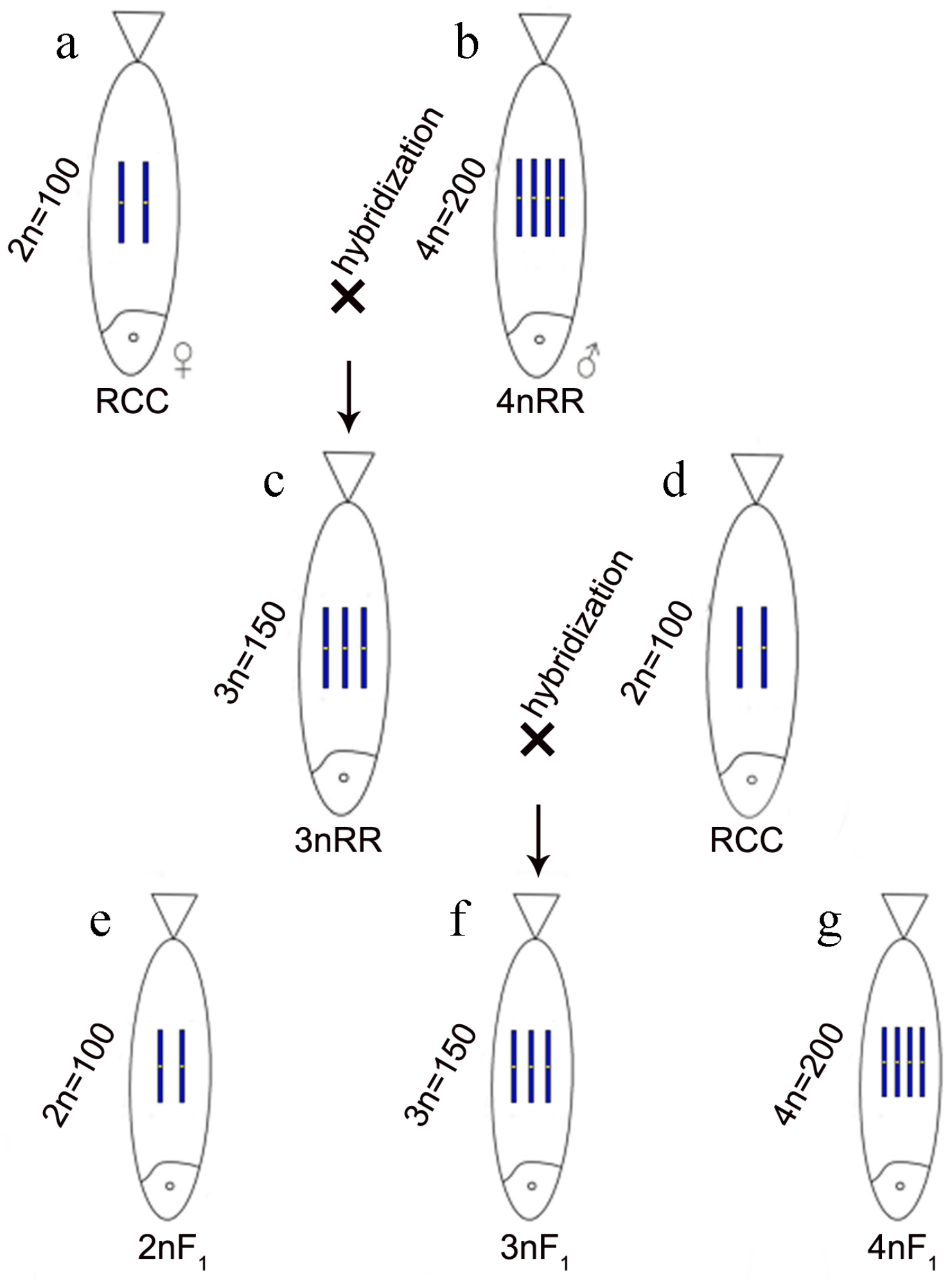

Figure 1

Formation of polyploid fish. 

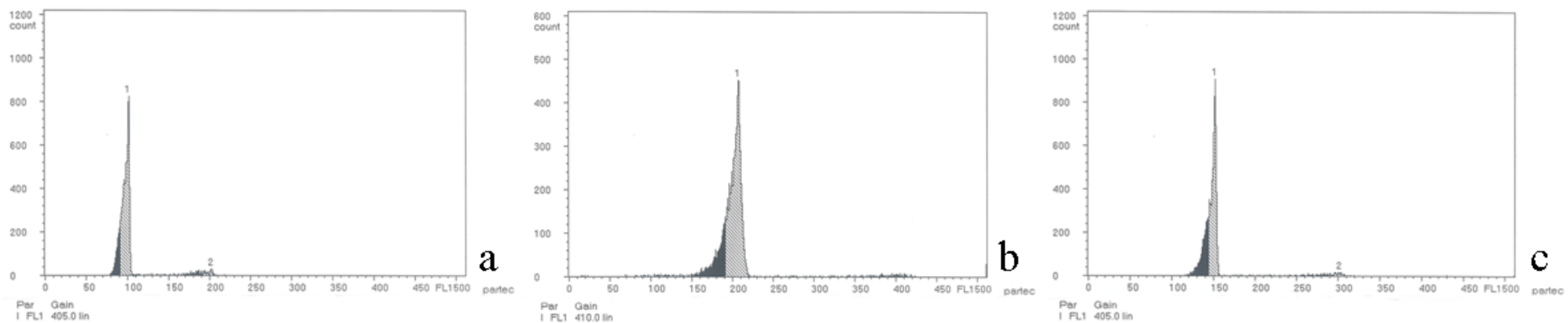

Figure 2

DNA-content flow-cytometry histograms of RCC (a), 4nRR (b) and 3nRR (c).

a
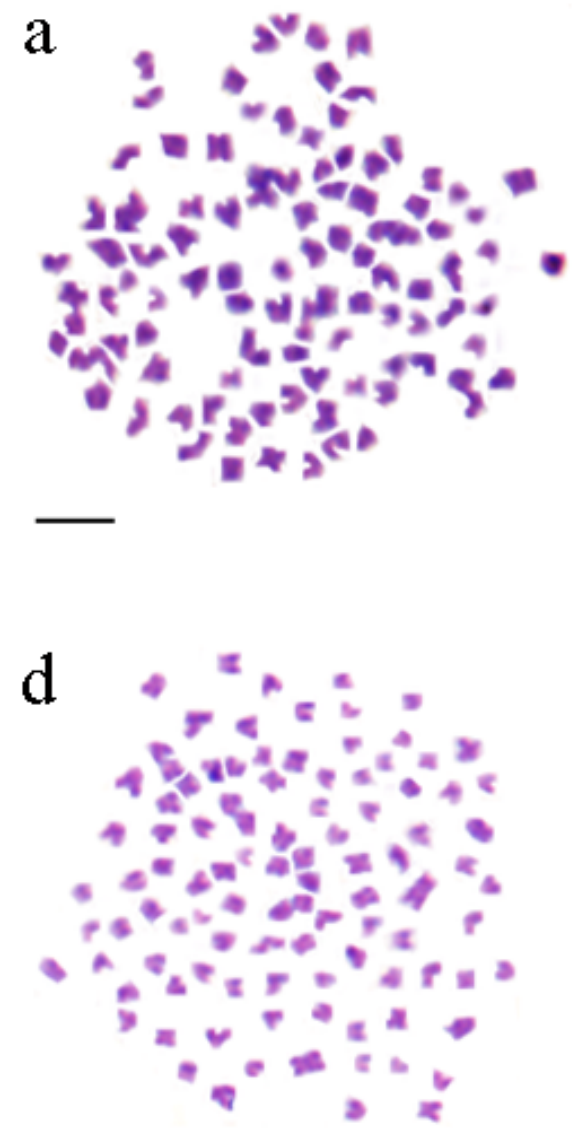

b

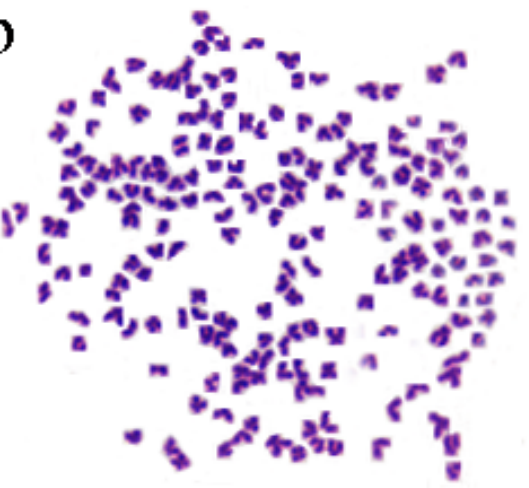

$\mathrm{e}$

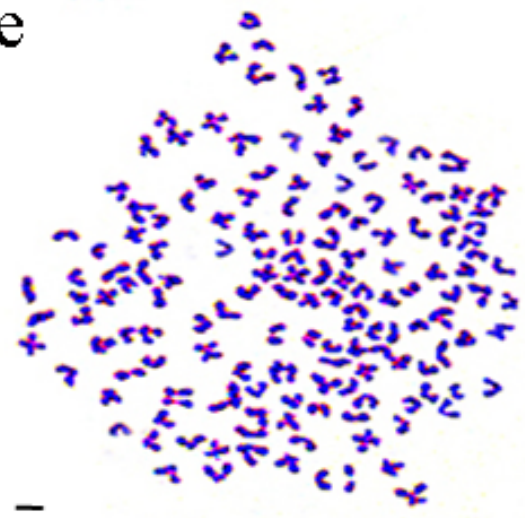

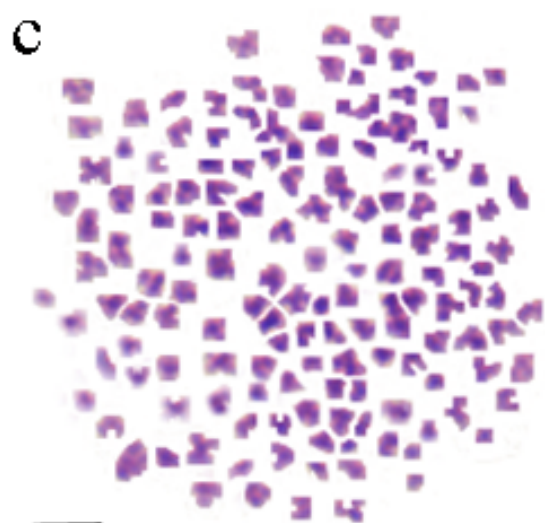

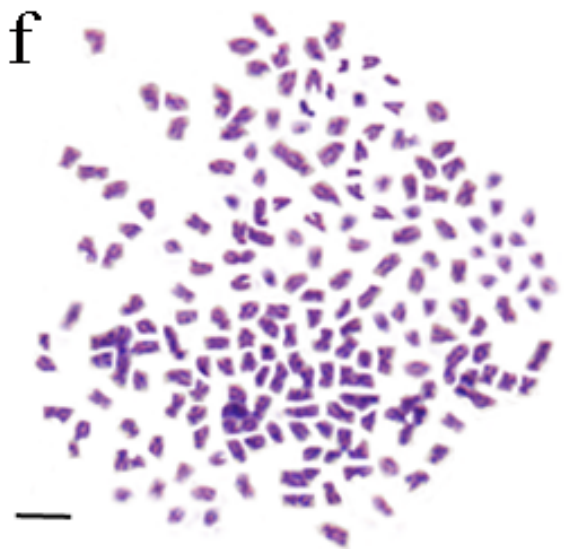

Figure 3

Chromosome spreads at metaphase in RCC, 4nRR, 3nRR, 2nF1, 3nF1 and 4nF1. a: The 100 chromosomes of RCC; b: The 200 chromosomes of $4 \mathrm{nRR}$; $\mathrm{c}$ : The 150 chromosomes of $3 \mathrm{nRR}$; $\mathrm{d}$ : The 100 chromosomes of 2nF1; e: The 150 chromosomes of 3nF1; f: The 200 chromosomes of $4 \mathrm{nF} 1$; bar $=5 \mu \mathrm{m}$. 

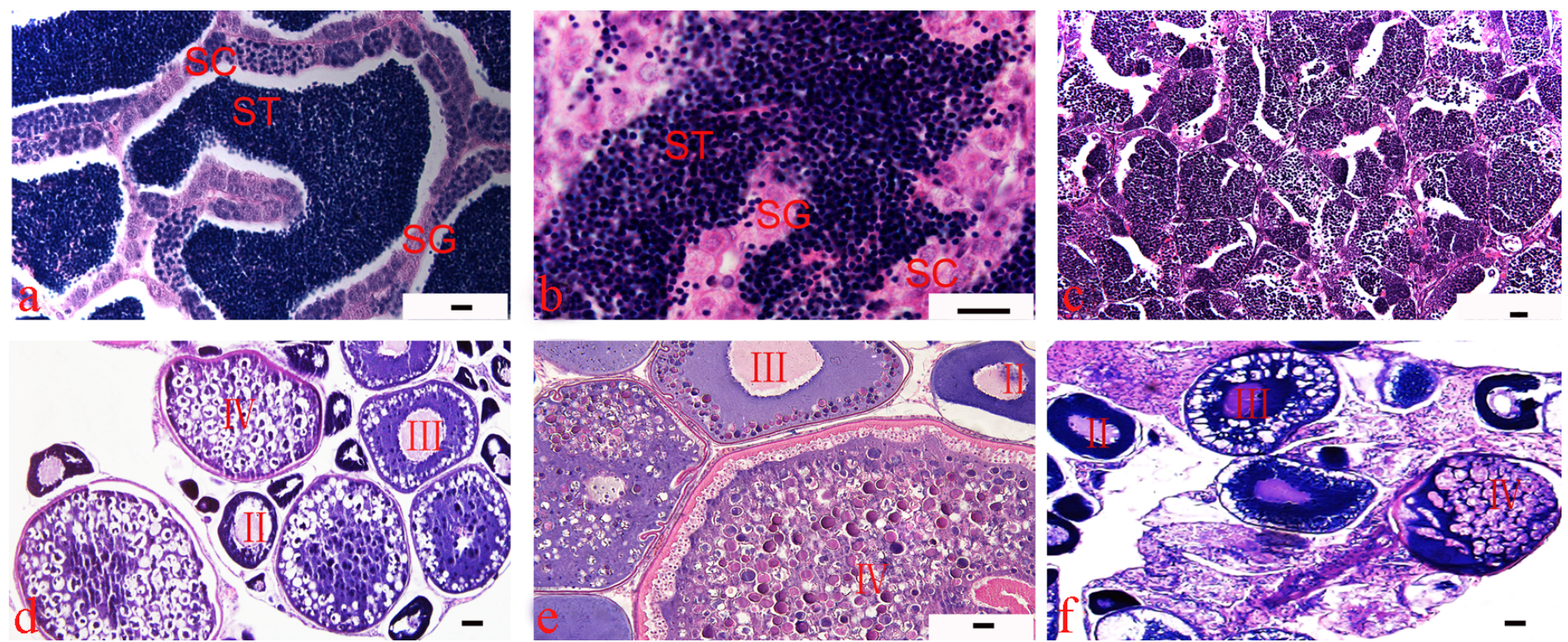

Figure 4

Micrographs of the testes and ovaries of RCC, 3nRR and 4nRR. a: Micrographs of testis from RCC; $b$ : Micrographs of testis from 4nRR; c: Micrographs of testis from 3nRR; d: Micrographs of ovary from RCC; e: Micrographs of ovary from 4nRR; f: Micrographs of ovary from 3nRR; SG: spermatogonia; SC:

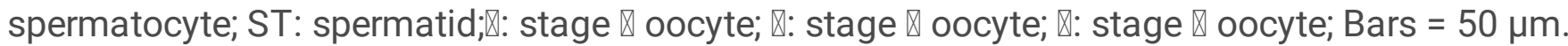
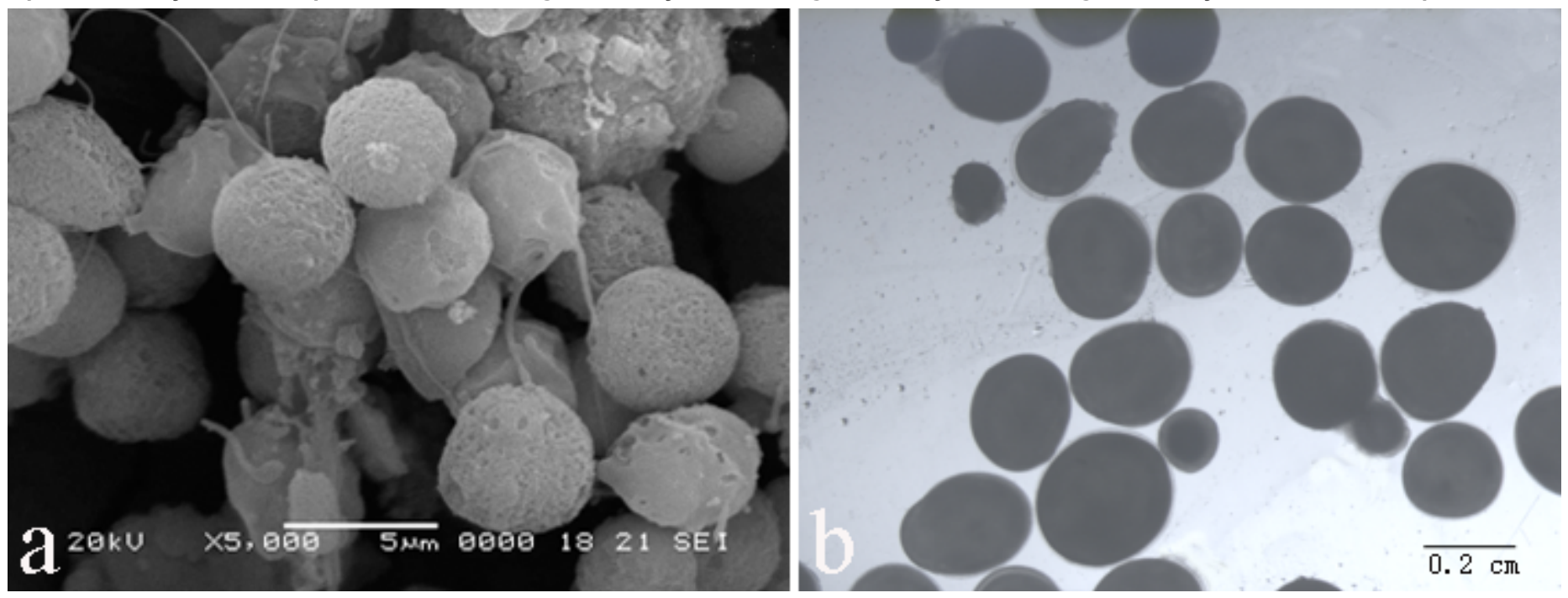

Figure 5

Spermatozoa and eggs of 3nRR. a: Abnormal spermatozoa produced by 3nRR males; b: Different sizes of eggs collected from 2-year-old 3nRR females. 

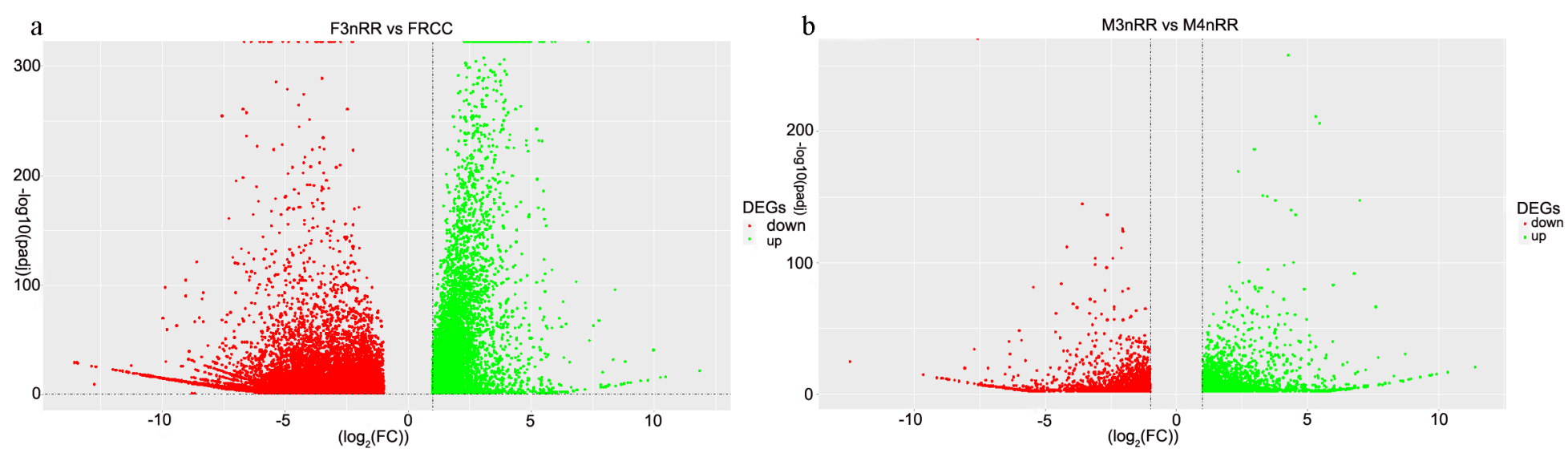

\section{Figure 6}

Volcano plot for gene differential expression. a: F3nRR vs FRCC; b: M3nRR vs M4nRR. Genes with FDR < 0.05 and ratio of FPKMs of the two samples $>2$ were considered to be differentially expressed genes. The red region shows significantly up-regulated genes, whereas the green region shows down-regulated genes

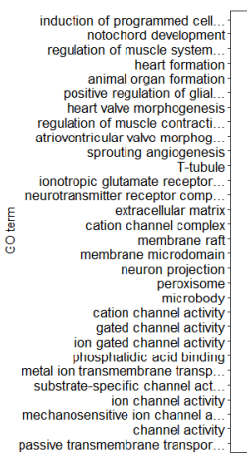

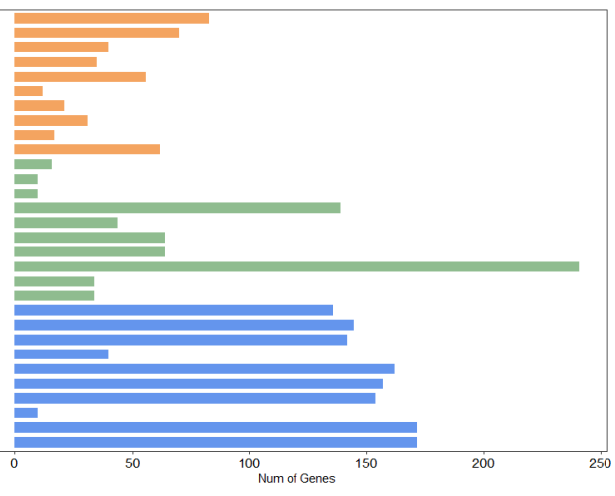

b

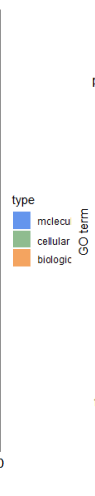

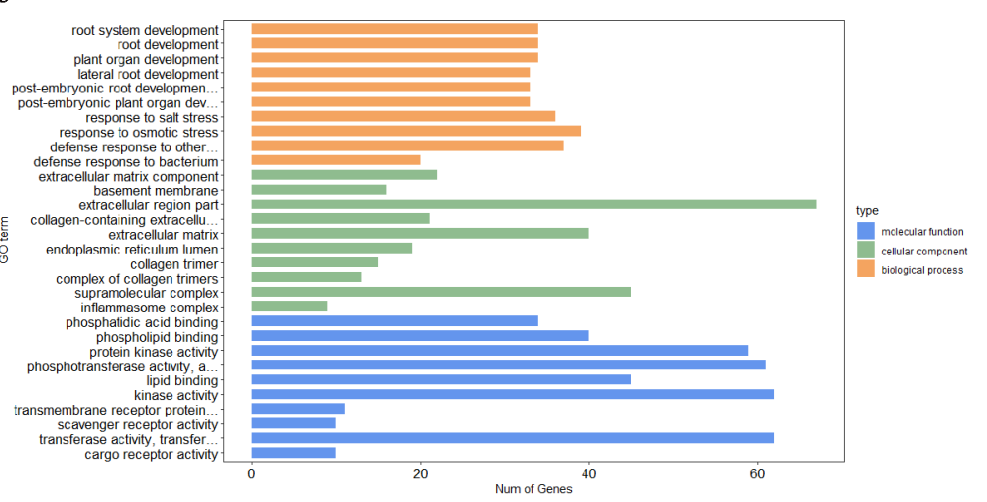

\section{Figure 7}

Gene Ontology (GO) functional classification of differentially expressed genes (DEGs). a: F3nRR vs FRCC; b: M3nRR vs M4nRR. The three terms are presented on the x-axis shows and the proportion of DEGs corresponding to each subcategory is presented on the $y$-axis.
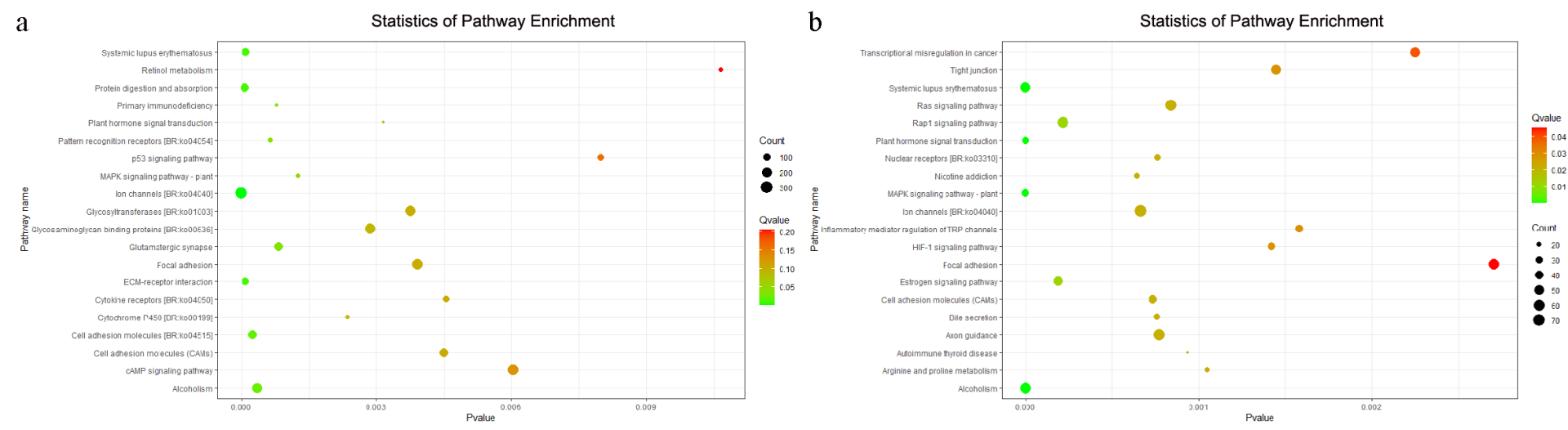

Figure 8 
Statistics of Kyoto Encyclopedia of Genes and Genomes (KEGG) pathway enrichment analysis of the functional significance of DEGs. a: F3nRR vs FRCC; $b$ : M3nRR vs M4nRR. The abscissa represents $P$ value, which decreases with increase in significance in enrichment level of differentially expressed genes in the pathway. The ordinate represents $\log 10$ ( $Q$ value), which increases with increase in significance of differentially expressed genes in the pathway.

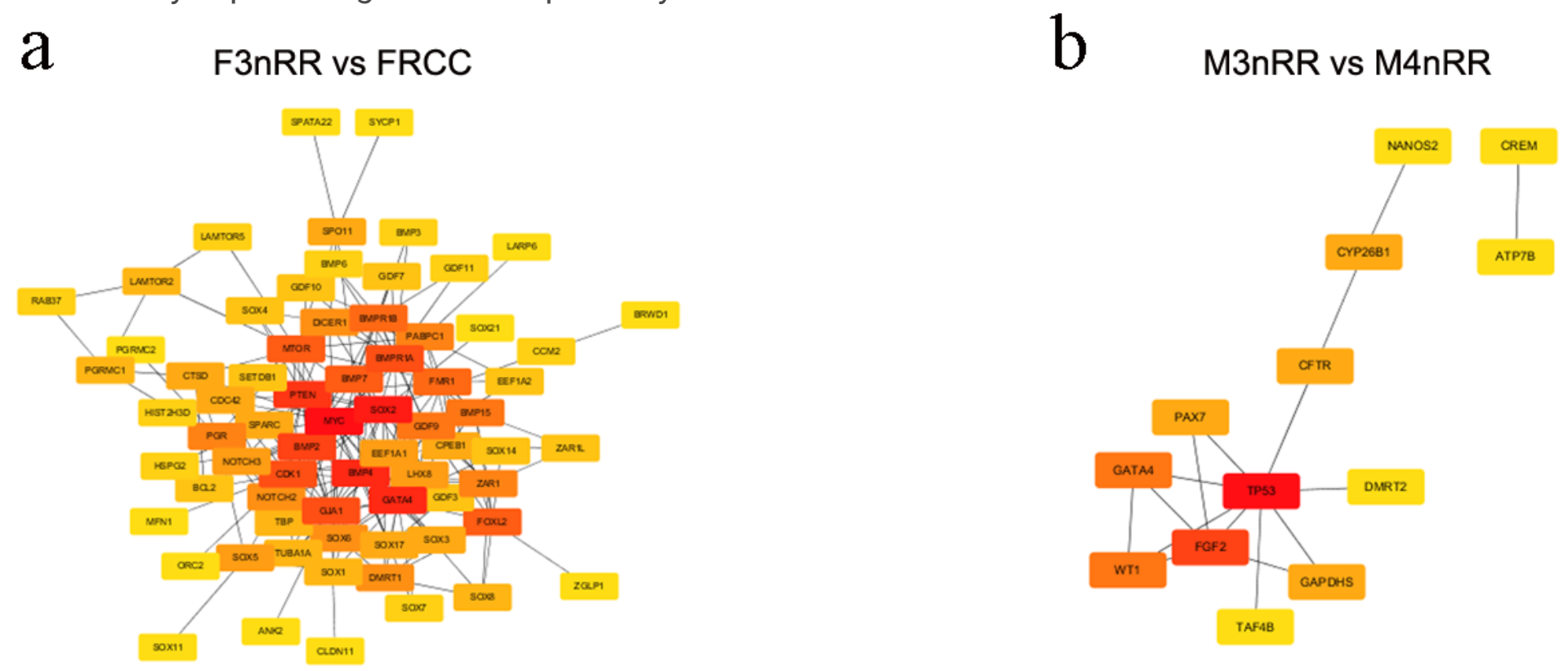

\section{Figure 9}

Protein-protein interaction network of fertility-related genes. a: F3nRR vs FRCC; b: M3nRR vs M4nRR. Genes with higher degree value are presented in deep red color.

a

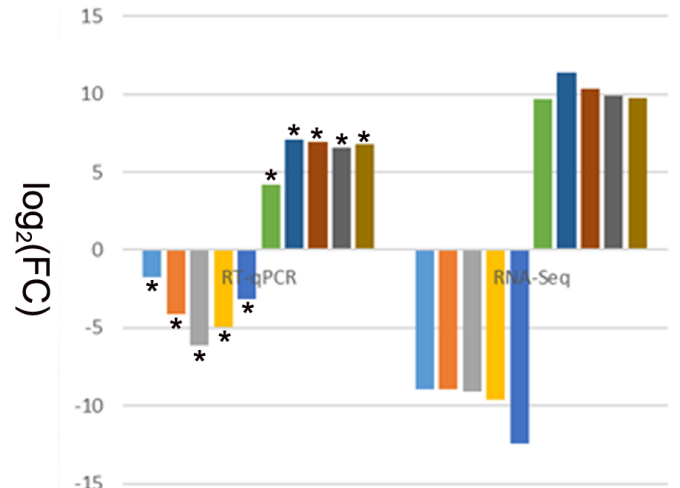

b
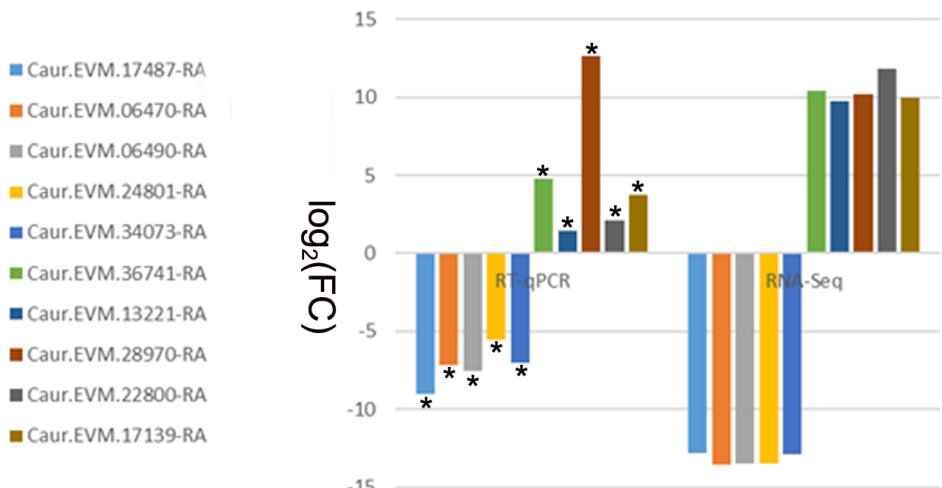

= Caur.EVM. 27390-RA

a Caur.EVM.24201-RA = Caur.EVM. 24200-RA = Caur.EVM.34835-RA n Caur.EVM.14945-RA n Caur.EVM.26856-RA a Caur.EVM.26636-RA a Caur.EVM.02475-RA a Caur.EVM.13777-RA a Caur.EVM.35979-RA

\section{Figure 10}

Comparison between RNA-seq and RT-qPCR results. a: F3nRR vs FRCC; b: M3nRR vs M4nRR. Significant differences are indicated: ${ }^{*} p<0.05$.

\section{Supplementary Files}


This is a list of supplementary files associated with this preprint. Click to download.

- Additionalfile1.docx

- Additionalfile2.xlsx

- Additionalfile3.xlsx

- Additionalfile4.xlsx

- Additionalfile5.xIsx

- Additionalfile6.xlsx

- Additionalfile7.xlsx

- Additionalfile8.docx

- Additionalfile1.docx

- Additionalfile2.xlsx

- Additionalfile3.xlsx

- Additionalfile4.xlsx

- Additionalfile5.xlsx

- Additionalfile6.xlsx

- Additionalfile7.xIsx

- Additionalfile8.docx 